\title{
Lack of Associations between XPC Gene Polymorphisms and Neuroblastoma Susceptibility in a Chinese Population
}

\author{
Jintao Zheng, ${ }^{1,2}$ Ruizhong Zhang, ${ }^{2}$ Jinhong Zhu, ${ }^{3}$ Fenghua Wang, ${ }^{2}$ Tianyou Yang, \\ Jing $\mathrm{He}^{2}$ and Huimin $\mathrm{Xia}^{1,2}$ \\ ${ }^{1}$ Department of Pediatric Surgery, Foshan Maternity and Children's Healthcare Hospital Affiliated to Southern Medical University, \\ Foshan, China \\ ${ }^{2}$ Department of Pediatric Surgery, Guangzhou Institute of Pediatrics, Guangzhou Women and Children's Medical Center, \\ Guangzhou Medical University, Guangzhou, Guangdong 510623, China \\ ${ }^{3}$ Molecular Epidemiology Laboratory and Department of Laboratory Medicine, Harbin Medical University Cancer Hospital, Harbin, \\ Heilongjiang 150040, China
}

Correspondence should be addressed to Jing He; hejing198374@gmail.com and Huimin Xia; xia-huimin@foxmail.com

Received 11 July 2016; Accepted 27 September 2016

Academic Editor: Hai-Feng Pan

Copyright (C) 2016 Jintao Zheng et al. This is an open access article distributed under the Creative Commons Attribution License, which permits unrestricted use, distribution, and reproduction in any medium, provided the original work is properly cited.

\begin{abstract}
Neuroblastoma is one of the most malignant solid tumors in infants and young children. No more than $40 \%$ of neuroblastoma patients can survive for longer than five years after it has been diagnosed. XPC protein is a pivotal factor that recognizes DNA damage and starts up the nucleotide excision repair (NER) in mammalian cells. This makes up the first group to defend against the cancer. Previous studies have identified that XPC gene polymorphisms were associated with various types of cancer. However, the associations between $X P C$ gene polymorphisms and neuroblastoma risk have not yet been studied. We investigated the associations between three XPC gene polymorphisms (rs2228001 A>C, rs2228000 C > T, and rs2229090 G>C) and neuroblastoma risk with 256 neuroblastoma patients and 531 healthy controls in a Chinese Han population. Odds ratios and 95\% confidence intervals were used to access the association between these three polymorphisms and neuroblastoma risk. No significant association was detected between these three polymorphisms and neuroblastoma risk in the overall analysis as well as in the stratification analysis. These results suggest that none of these three polymorphisms may be associated with the risk of neuroblastoma in the Chinese Han population.
\end{abstract}

\section{Introduction}

Neuroblastoma originates in primitive neural crest cells of the adrenal medulla or sympathetic ganglia. It is one of the most malignant solid tumors in infants and young children, in particular, accounting for $7 \%-10 \%$ of childhood tumors. The tumor is found to primarily locate in the retroperitoneal parts (approximately 60\%) and secondly in the mediastinum, pelvis, and cervical sympathetic ganglion. The rest, about $12 \%$, associates with other malformations [1-3]. The morbidity of neuroblastoma in the live births is about 7.7 cases per million in China [4]. Despite the application of multimodality treatment including surgery, chemotherapy, and radiotherapy, no more than $40 \%$ of neuroblastoma patients could survive for longer than five years after diagnosis. Until now, the etiology of neuroblastoma remains largely unclear $[5,6]$.

Genome-wide association study (GWAS) has been used as a convenient and powerful approach for examining inherited genetic variations in relation to human complex disease, such as cancer susceptibility [7, 8]. In 2008, Maris et al. [9] used the GWAS approach to prove that three single nucleotide polymorphisms (SNPs) in CASC15 gene at chromosome 6p22 (rs6939340, rs4712653, and rs9295536) were highly associated with neuroblastoma susceptibility in European descents. Since then, lots of SNPs in specific chromosomal regions have been identified by the GWAS approach. 
Several gene polymorphisms are significantly associated with the risk of neuroblastoma, such as LIN28B [10], HACE1 [10], BARD1 [11], LMO1 [12], HSD17B12 [13], DDX4 [13], IL31RA [13], and DUSP12 [13], which have been discovered by means of GWAS. Besides, several genes, such as FAS [14], FASL [14], NEFL [15], and TGFBR3L [16], have been discovered by candidate gene approach.

Nucleotide excision repair (NER) can preserve the hereditary integrity and stability of genomic DNA through eliminating DNA damage generated by exogenous agents (i.e., mutagenic and carcinogenic substances) and photoproducts caused by sunlight exposure $[17,18]$. In humans, hereditary defects of genomic DNA in NER are involved in several autosomal recessive diseases. Xeroderma pigmentosum (XP) is characterized by a strong predisposition to skin carcinomas. However, it was not until 1968 that Cleaver [19] identified that XP could result from the defects in NER. NER is a complex biochemical process that involves hundreds of components in human cells, including the seven XP-related genes (XPA, $X P B, X P C, X P D, X P E, X P F$, and $X P G)$. XPC protein is an important DNA damage recognition protein and an initiator of the NER process that defends against cancer [20-25].

Some XPC gene SNPs have been identified to be implicated in melanoma [26], colorectal cancer [27, 28], prostate cancer [29], hepatocellular cancer [30], lung cancer [31], and gastric cancer [32]. However, the association between XPC gene polymorphisms and neuroblastoma risk has not been studied. In view of this, we investigated the relationship between XPC gene polymorphisms (rs2228001 $\mathrm{A}>\mathrm{C}$, rs2228000 C>T, and rs2229090 G>C) and neuroblastoma susceptibility in a Chinese Han population making use of a total of 787 participants (256 cases and 531 controls).

\section{Materials and Methods}

2.1. Study Subjects. A total of 256 newly diagnosed and histopathologically confirmed neuroblastoma cases were recruited from the Guangzhou Women and Children's Medical Center between February 2010 and November 2015 [3335]. During the same period, 531 healthy children were randomly selected as the age- and gender-matched controls after receiving routine physical examination [35-37]. Both the cases and the controls were unassociated ethnic Chinese Han individuals. The study was granted permission by the Institutional Review Board of Guangzhou Women and Children's Medical Center. Demographic factors and medical histories were gathered through the structured questionnaires. Written informed consent was received from the guardians of each child participant. Each participant donated $2 \mathrm{~mL}$ of blood for genomic DNA extraction.

2.2. Polymorphism Analysis. The selection criteria were described previously, in terms of the minor allele frequency, putative functional potentials, and low linkage disequilibrium [38]. Based on the criteria, three potentially functional SNPs (rs2228001 A >C, rs2228000 C>T, and rs2229090 G>C) were selected. s2228001 A>C (Lys939Gln) and rs2228000 C>T (Val499Arg) are nonsynonymous SNPs and they have been widely investigated in various types of cancer. XPC rs2229090 G>C is $3^{\prime} \mathrm{UTR}$ polymorphism within miRNA binding site. Genomic DNA was extracted from $2 \mathrm{~mL}$ of peripheral blood sample using the TIANamp Blood DNA Kit (TianGen Biotech Co. Ltd., Beijing, China) following the manufacturer's instructions. Qualified DNA samples were diluted to $10 \mathrm{ng} / \mu \mathrm{L}$ and loaded in 96-well plates. Then, these three SNPs were genotyped using Taqman real-time PCR method as described previously $[39,40]$.

2.3. Statistical Analysis. $\chi^{2}$ test was used to evaluate the differences in the frequency distributions of the demographics and genotypes between the neuroblastoma cases and the controls. Hardy-Weinberg equilibrium (HWE) was tested in the controls by the goodness-of-fit chi-squared test. Odds ratios (ORs) and 95\% confidence intervals (CIs) were used to assess the correlations between the three polymorphisms and neuroblastoma susceptibility with the unconditional multivariate logistic regression analysis. $P$ value $<0.05$ was considered as statistically significant. All statistical tests were two-sided and analyzed using SAS software (version 9.1; SAS Institute, Cary, NC).

\section{Results}

3.1. Frequency Distribution of Selected Characteristics. As displayed in Supplemental Table 1 in Supplementary Material available online at http://dx.doi.org/10.1155/2016/2932049, the average age of the cases was $30.87 \pm 26.45$ months and $29.73 \pm 24.86$ months for the controls. No significant differences were observed in terms of age $(P=0.239)$ and gender $(P=0.333)$ between the case and the control groups. According to the INSS standard [3], 54, 65, 44, 77, and 9 patients developed clinical stages I, II, III, and IV and $4 \mathrm{~s}$ neuroblastoma, respectively. Among these cases, 46 lesions occurred in adrenal gland, 87 in retroperitoneal region, and 90 in mediastinum.

3.2. XPC Gene Polymorphisms and Neuroblastoma Susceptibility. In the current study, 253 cases and 531 controls were successfully genotyped. The genotype frequencies of the three polymorphisms are shown in Table 1 . The observed genotype frequencies of the three SNPs were in accordance with HWE in the control subjects $(P=0.948$ for $\mathrm{rs} 2228001 \mathrm{~A}>\mathrm{C}$ polymorphism, $P=0.988$ for $\mathrm{rs} 2228000 \mathrm{C}>\mathrm{T}$ polymorphism, and $P=0.994$ for rs2229090 G>C polymorphism). There is no significant association between rs2228001 $\mathrm{A}>\mathrm{C}$ polymorphism and neuroblastoma susceptibility. Similar results were found for rs2228000 C>T and rs2229090 G>C polymorphisms.

3.3. Stratification Analysis of XPC Gene Polymorphisms with Neuroblastoma Susceptibility. Stratified analyses were conducted regarding age, gender, sites of origin, and clinical stages to assess the association of the three selected polymorphisms with the risk of neuroblastoma (Table 2). However, no significant association was identified for any of the three polymorphisms. 


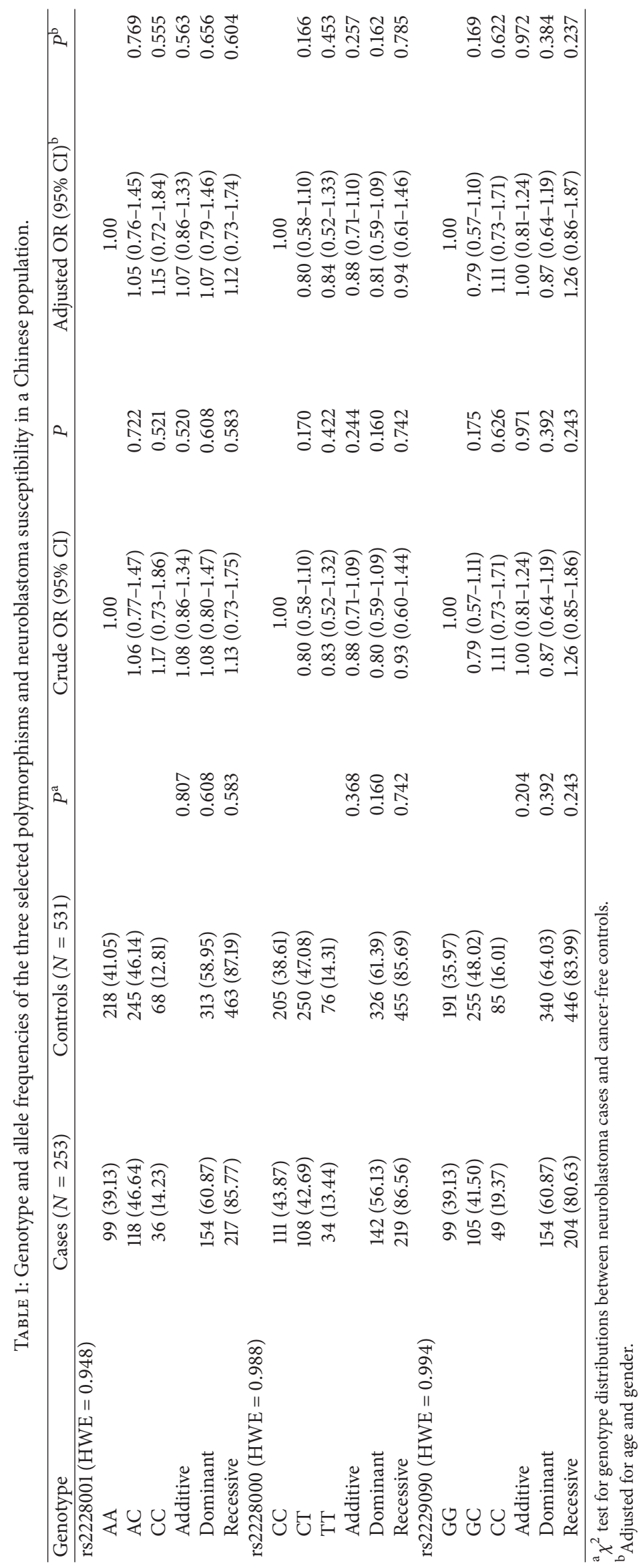




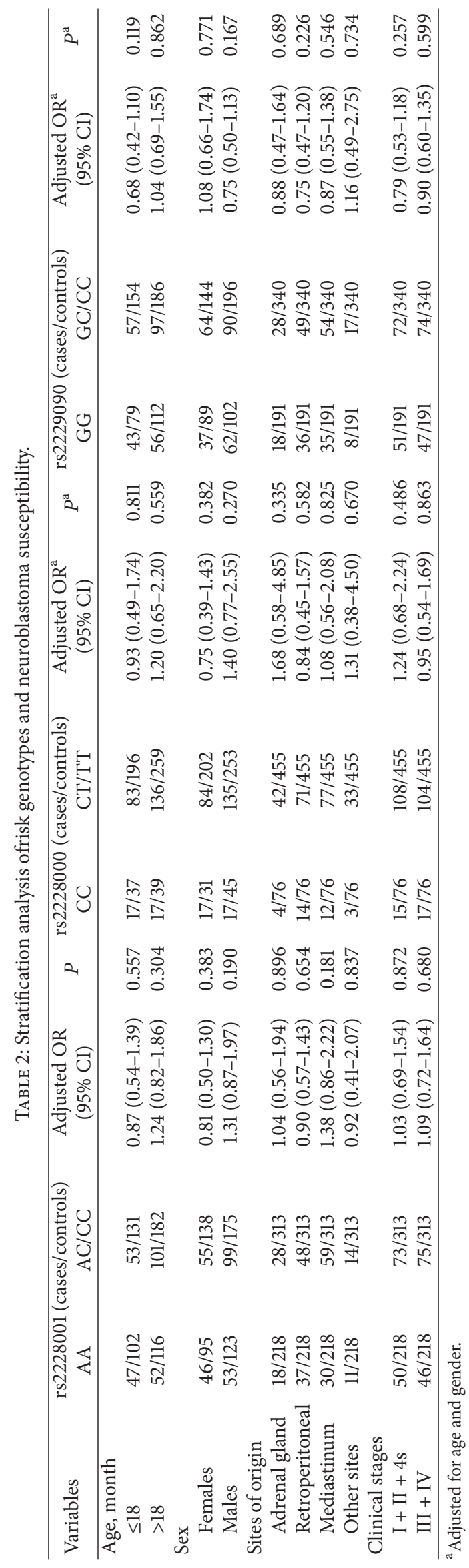




\section{Discussion}

In this hospital-based study comprising 256 cases and 531 controls, none of the three XPC gene polymorphisms was associated with neuroblastoma risk when compared to the reference genotypes. To the best of our knowledge, this is the first investigation looking into the relationship between XPC gene polymorphisms and neuroblastoma susceptibility in a Chinese Han population.

XPC gene (http://www.ncbi.nlm.nih.gov/gene/7508) is located on chromosome 3p25.1 with 18 exons, which encodes a component of the NER. XPC plays a distinctively vital role in the early stages of global genome NER. XPC and UV excision repair protein RAD23 homolog B (HR23B) form the XPC-HR23B complex, which recognizes DNA damage and initiates NER in mammalian cells, thereby protecting against cancer [20-25, 41, 42].

$X P C$ gene polymorphisms are involved in the different types of cancer. Among them, rs2228001 A $>C$ and rs2228000 C $>$ T were widely investigated. Paszkowska-Szczur et al. [26] genotyped 714 melanoma cases and 1841 healthy controls to evaluate the relationship between 94 SNPs within the seven $X P$ genes $(X P A-X P G)$ and the melanoma risk in a Polish population. They found that XPC rs2228000 CT and TT genotypes were significantly associated with decreased melanoma risk when compared with the reference genotype. Moreover, they observed that rs2228000 CT genotype and rs2228000 TT genotype were associated with decreased colorectal cancer risk compared to the CC genotype in 758 colorectal cancer patients and the same number of controls [27]. In a study conducted in Malaysia with 255 colorectal cancer patients and 255 controls, Ahmad Aizat et al. [28] found that XPC gene rs 2228000 GG genotype was associated with an increased colorectal cancer risk. However, there is no previous study investigating the association between XPC gene polymorphisms and neuroblastoma susceptibility [43]. Our study fits the niche and it found no association between the three SNPs of XPC gene and neuroblastoma. However, the negative results might result from the relatively small sample size in this study, although it was the largest study regarding the Chinese children to date.

\section{Conclusions}

In summary, all the three XPC gene polymorphisms (rs2228001 A>C, rs2228000 C > T, and rs2229090 G>C) may not associate with neuroblastoma risk in the Chinese Han population. However, further studies with larger sample size and different ethnicities should be carried out to verify our results.
Abbreviations
GWAS: Genome-wide association study
SNP: Single nucleotide polymorphism
XPC: Xeroderma pigmentosum complementation C
NER: Nucleotide excision repair pathway
XP: Xeroderma pigmentosum
OR: Odds ratio
CI: Confidence interval.

\section{Competing Interests}

The authors declare that there are no competing interests regarding the publication of this paper.

\section{Authors' Contributions}

All authors contributed significantly to this work. Jintao Zheng, Ruizhong Zhang, Fenghua Wang, and Tianyou Yang performed the research study and collected the data; Jing He and Jintao Zheng analyzed the data; Jing He and Huimin Xia designed the research study; Jintao Zheng, Jinhong Zhu, and Jing He wrote the paper; and Jing He prepared all the tables. All authors reviewed the manuscript. In addition, all authors approved the final draft.

\section{Acknowledgments}

This work was supported by the grant of State Clinical Key Specialty Construction Project (Pediatric Surgery) 2013 (no. GJLCZD1301), the grant of Clinical Medicine Research and Transformation Center of Brain Injury in Premature Infant in Guangzhou (no. 520101-2150092), and the National Natural Science Foundation of China (no. 81502046). The authors thank Yanlu Tong and Hezhen Wang for their assistance in DNA extraction and medical histories information collection.

\section{References}

[1] M. A. Smith, N. L. Seibel, S. F. Altekruse et al., "Outcomes for children and adolescents with cancer: challenges for the twentyfirst century," Journal of Clinical Oncology, vol. 28, no. 15, pp. 2625-2634, 2010.

[2] J. M. Maris, M. D. Hogarty, R. Bagatell, and S. L. Cohn, "Neuroblastoma," The Lancet, vol. 369, no. 9579, pp. 2106-2120, 2007.

[3] G. M. Brodeur, J. Pritchard, F. Berthold et al., "Revisions of the international criteria for neuroblastoma diagnosis, staging, and response to treatment," Journal of Clinical Oncology, vol. 11, no. 8, pp. 1466-1477, 1993.

[4] P.-P. Bao, K. Li, C.-X. Wu et al., "Recent incidences and trends of childhood malignant solid tumors in Shanghai, 2002-2010," Zhonghua Er Ke Za Zhi, vol. 51, no. 4, pp. 288-294, 2013.

[5] F. Berthold, J. Boos, S. Burdach et al., "Myeloablative megatherapy with autologous stem-cell rescue versus oral maintenance chemotherapy as consolidation treatment in patients with highrisk neuroblastoma: a randomised controlled trial," The Lancet Oncology, vol. 6, no. 9, pp. 649-658, 2005.

[6] J. M. Maris, "Recent advances in neuroblastoma," The New England Journal of Medicine, vol. 362, no. 23, pp. 2154-2211, 2010.

[7] K. A. Frazer, S. S. Murray, N. J. Schork, and E. J. Topol, "Human genetic variation and its contribution to complex traits," Nature Reviews Genetics, vol. 10, no. 4, pp. 241-251, 2009.

[8] T. A. Manolio, "Genomewide association studies and assessment of the risk of disease," The New England Journal of Medicine, vol. 363, no. 2, pp. 166-176, 2010.

[9] J. M. Maris, Y. P. Mosse, J. P. Bradfield et al., "Chromosome 6p22 locus associated with clinically aggressive neuroblastoma," The 
New England Journal of Medicine, vol. 358, no. 24, pp. 25852593, 2008.

[10] S. J. Diskin, M. Capasso, R. W. Schnepp et al., "Common variation at $6 \mathrm{q} 16$ within HACE1 and LIN28B influences susceptibility to neuroblastoma," Nature Genetics, vol. 44, no. 10, pp. 11261130, 2012.

[11] M. Capasso, M. Devoto, C. Hou et al., "Common variations in BARD1 influence susceptibility to high-risk neuroblastoma," Nature Genetics, vol. 41, no. 6, pp. 718-723, 2009.

[12] K. Wang, S. J. Diskin, H. Zhang et al., "Integrative genomics identifies LMO1 as a neuroblastoma oncogene," Nature, vol. 469, no. 7329, pp. 216-220, 2011.

[13] L. B. Nguyễn, S. J. Diskin, M. Capasso et al., "Phenotype restricted genome-wide association study using a gene-centric approach identifies three low-risk neuroblastoma susceptibility loci," PLoS Genetics, vol. 7, no. 3, Article ID e1002026, 2011.

[14] W. Han, Y. Zhou, R. Zhong et al., "Functional polymorphisms in FAS/FASL system increase the risk of neuroblastoma in Chinese population," PLoS ONE, vol. 8, no. 8, Article ID e71656, 2013.

[15] M. Capasso, S. Diskin, F. Cimmino et al., "Common genetic variants in NEFL influence gene expression and Neuroblastoma risk," Cancer Research, vol. 74, no. 23, pp. 6913-6924, 2014.

[16] Y. Jin, H. Wang, W. Han et al., "Single nucleotide polymorphism rs11669203 in TGFBR3L is associated with the risk of neuroblastoma in a Chinese population," Tumor Biology, vol. 37, no. 3, pp. 3739-3747, 2016.

[17] R. D. Wood, M. Mitchell, J. Sgouros, and T. Lindahl, "Human DNA repair genes," Science, vol. 291, no. 5507, pp. 1284-1289, 2001.

[18] E. C. Friedberg, "How nucleotide excision repair protects against cancer," Nature Reviews Cancer, vol. 1, no. 1, pp. 22-33, 2001.

[19] J. E. Cleaver, "Defective repair replication of DNA in xeroderma pigmentosum," Nature, vol. 218, no. 5142, pp. 652-656, 1968.

[20] T. Riedl, F. Hanaoka, and J.-M. Egly, "The comings and goings of nucleotide excision repair factors on damaged DNA," EMBO Journal, vol. 22, no. 19, pp. 5293-5303, 2003.

[21] M. Volker, M. J. Moné, P. Karmakar et al., "Sequential assembly of the nucleotide excision repair factors in vivo," Molecular Cell, vol. 8, no. 1, pp. 213-224, 2001.

[22] J. H. J. Hoeijmakers, "Genome maintenance mechanisms for preventing cancer," Nature, vol. 411, no. 6835, pp. 366-374, 2001.

[23] J. De Boer and J. H. J. Hoeijmakers, "Nucleotide excision repair and human syndromes," Carcinogenesis, vol. 21, no. 3, pp. 453460, 2000.

[24] W. L. de Laat, N. G. J. Jaspers, and J. H. J. Hoeijmakers, "Molecular mechanism of nucleotide excision repair," Genes \& Development, vol. 13, no. 7, pp. 768-785, 1999.

[25] R. D. Wood, "Nucleotide excision repair in mammalian cells," Journal of Biological Chemistry, vol. 272, no. 38, pp. 2346523468, 1997.

[26] K. Paszkowska-Szczur, R. J. Scott, P. Serrano-Fernandez et al., "Xeroderma pigmentosum genes and melanoma risk," International Journal of Cancer, vol. 133, no. 5, pp. 1094-1100, 2013.

[27] K. Paszkowska-Szczur, R. J. Scott, B. Górski et al., "Polymorphisms in nucleotide excision repair genes and susceptibility to colorectal cancer in the Polish population," Molecular Biology Reports, vol. 42, no. 3, pp. 755-764, 2015.

[28] A. A. Ahmad Aizat, M. S. Siti Nurfatimah, M. M. Aminudin, and R. Ankathil, "XPC Lys939Gln polymorphism, smoking and risk of sporadic colorectal cancer among Malaysians," World Journal of Gastroenterology, vol. 19, no. 23, pp. 3623-3628, 2013.

[29] Y. Yoshino, S. Takeuchi, T. Katoh, and Y. Kuroda, "XPC intronl1 C/A polymorphism as a risk factor for prostate cancer," Environmental Health and Preventive Medicine, vol. 21, no. 2, pp. 100 104, 2016.

[30] B. Wang, Q. Xu, H. W. Yang, L. P. Sun, and Y. Yuan, "The association of six polymorphisms of five genes involved in three steps of nucleotide excision repair pathways with hepatocellular cancer risk," Oncotarget, vol. 7, no. 15, pp. 20357-20367, 2016.

[31] C. Mei, M. Hou, S. Guo et al., "Polymorphisms in DNA repair genes of XRCC1, XPA, XPC, XPD and associations with lung cancer risk in Chinese people," Thoracic Cancer, vol. 5, no. 3, pp. 232-242, 2014.

[32] J. Liu, L. Sun, Q. Xu et al., "Association of nucleotide excision repair pathway gene polymorphisms with gastric cancer and atrophic gastritis risks," Oncotarget, vol. 7, no. 6, pp. 6972-6983, 2016.

[33] J. He, F. Wang, J. Zhu et al., "Association of potentially functional variants in the XPG gene with neuroblastoma risk in a Chinese population," Journal of Cellular and Molecular Medicine, vol. 20, pp. 1481-1490, 2016.

[34] J. He, T. Yang, R. Zhang et al., "Potentially functional polymorphisms in the LIN28B gene contribute to neuroblastoma susceptibility in Chinese children," Journal of Cellular and Molecular Medicine, vol. 20, no. 8, pp. 1534-1541, 2016.

[35] J. He, W. Zhong, J. Zeng et al., "LMO1 gene polymorphisms contribute to decreased neuroblastoma susceptibility in a Southern Chinese population," Oncotarget, vol. 7, no. 16, pp. 22770-22778, 2016.

[36] R. Zhang, Y. Zou, J. Zhu et al., "The association between GWASidentified BARD1 gene SNPS and neuroblastoma susceptibility in a Southern Chinese population," International Journal of Medical Sciences, vol. 13, no. 2, pp. 133-138, 2016.

[37] J. He, R. Zhang, Y. Zou et al., "Evaluation of GWAS-identified SNPs at 6p22 with neuroblastoma susceptibility in a Chinese population," Tumor Biology, vol. 37, no. 2, pp. 1635-1639, 2016.

[38] R. X. Hua, Z. J. Zhuo, G. P. Shen et al., "Polymorphisms in the XPC gene and gastric cancer susceptibility in a Southern Chinese population," Journal of OncoTargets and Therapy, vol. 9, pp. 5513-5519, 2016.

[39] J. He, L.-X. Qiu, M.-Y. Wang et al., "Polymorphisms in the XPG gene and risk of gastric cancer in Chinese populations," Human Genetics, vol. 131, no. 7, pp. 1235-1244, 2012.

[40] J. He, M.-Y. Wang, L.-X. Qiu et al., "Genetic variations of mTORCl genes and risk of gastric cancer in an Eastern Chinese population," Molecular Carcinogenesis, vol. 52, supplement 1, pp. E70-E79, 2013.

[41] M. Akita, Y.-S. Tak, T. Shimura et al., "SUMOylation of xeroderma pigmentosum group $\mathrm{C}$ protein regulates DNA damage recognition during nucleotide excision repair," Scientific Reports, vol. 5, Article ID 10984, 2015.

[42] K. Sugasawa, J. M. Y. Ng, C. Masutani et al., "Xeroderma pigmentosum group $\mathrm{C}$ protein complex is the initiator of global genome nucleotide excision repair," Molecular Cell, vol. 2, no. 2, pp. 223-232, 1998.

[43] J. He, T.-Y. Shi, M.-L. Zhu, M.-Y. Wang, Q.-X. Li, and Q.-Y. Wei, "Associations of Lys939Gln and Ala499Val polymorphisms of the XPC gene with cancer susceptibility: a meta-analysis," International Journal of Cancer, vol. 133, no. 8, pp. 1765-1775, 2013. 


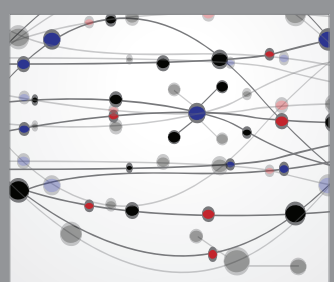

The Scientific World Journal
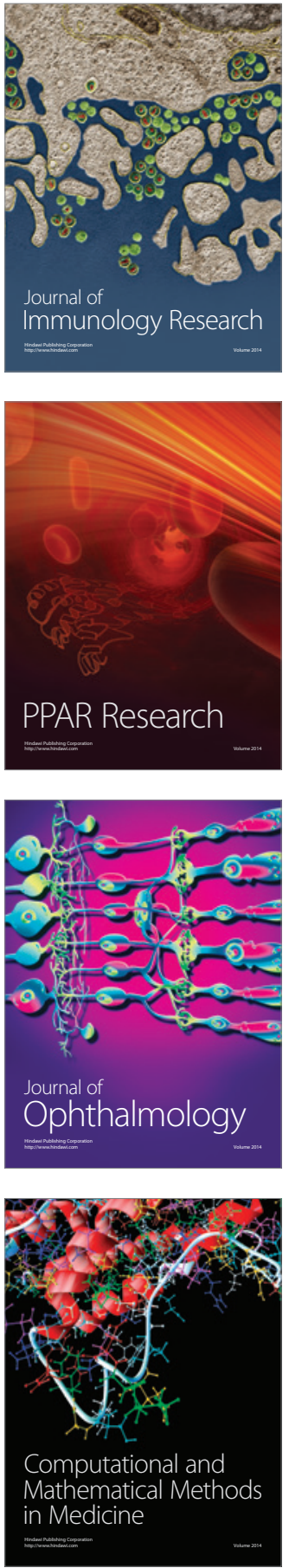

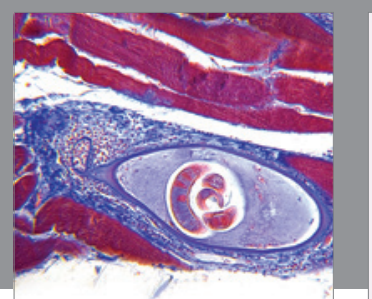

Gastroenterology Research and Practice

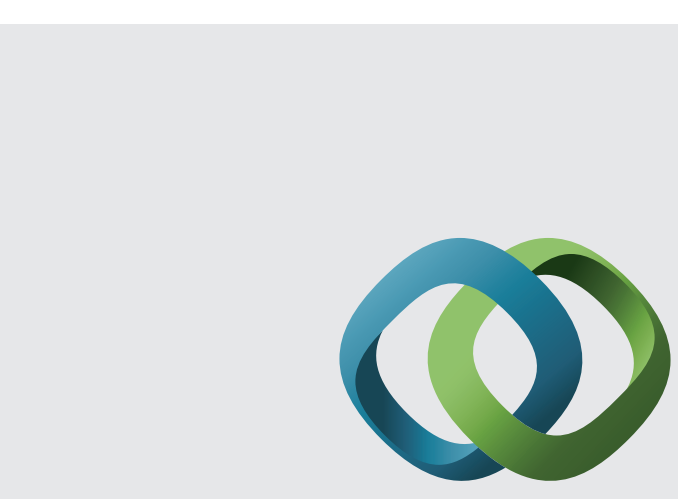

\section{Hindawi}

Submit your manuscripts at

http://www.hindawi.com
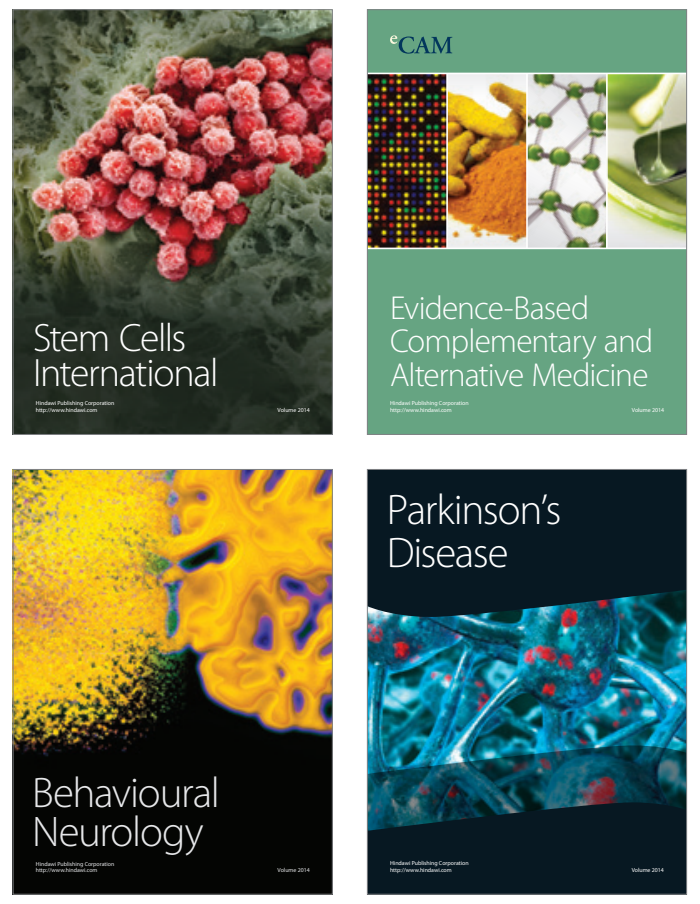
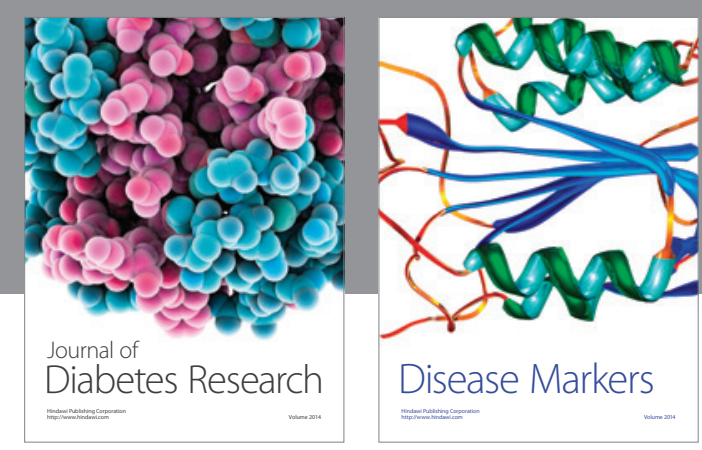

Disease Markers
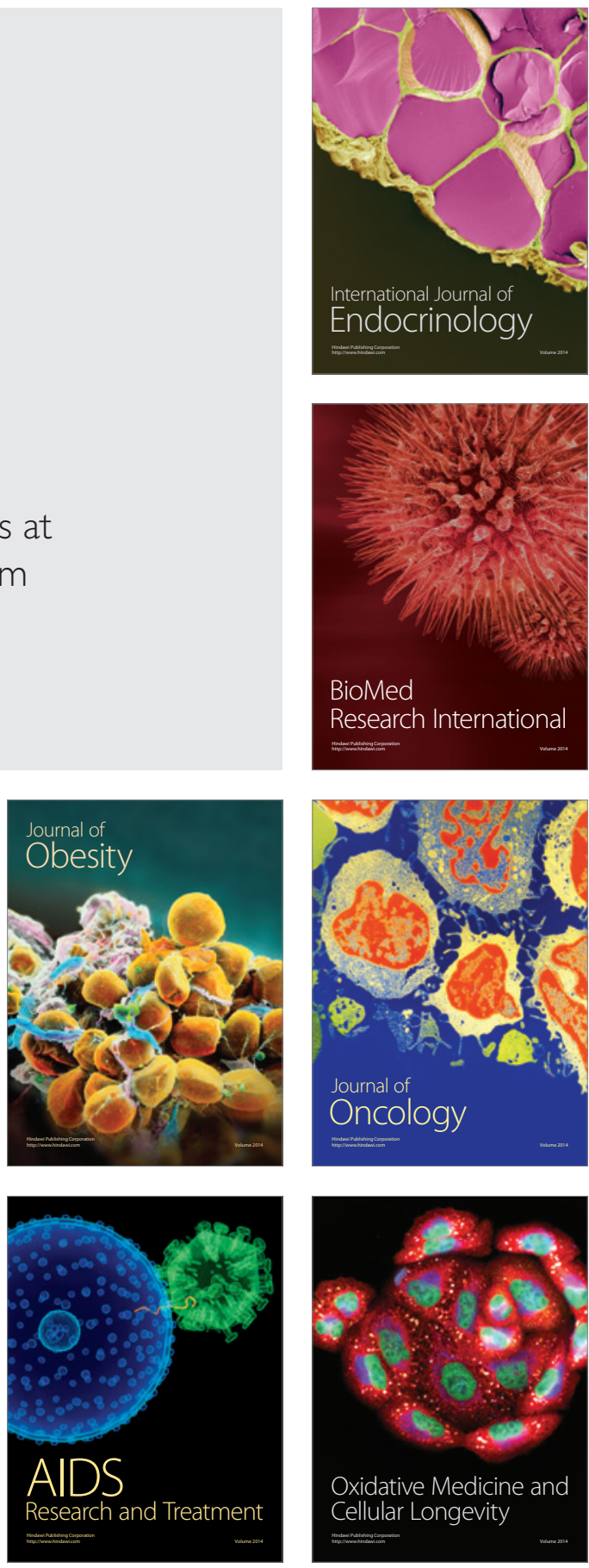